\title{
Influence of a Van Hove singularity on the electronic specific heat of high- $\mathrm{T}_{\mathrm{c}}$ superconductors
}

\author{
S. Dorbolo ', M. Houssa ${ }^{2, *}$, M. Ausloos ${ }^{3}$ \\ S.U.P.R.A.S., Institut de Physique B5. Université de Liège, B-4000 Liège, Belgium
}

Received 20 December 1995; revised manuscript received 18 March 1996

\begin{abstract}
We study the influence of a logarithmic Van Hove singularity on the electronic specific heat $C_{\mathrm{e}}$ of a 2D superconductor.

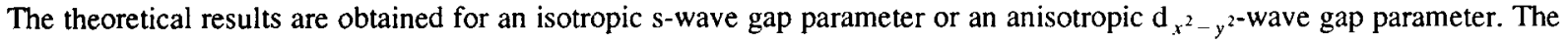
specific heat jump at $T_{c}$ observed in high- $\mathrm{T}_{\mathrm{c}}$ superconductors can be reproduced by considering both gap parameter symmetries. However the very low temperature behavior of $C_{\mathrm{e}}$ as observed in a single crystal of $\mathrm{YBa}_{2} \mathrm{Cu}_{3} \mathrm{O}_{7-\delta}$ is only consistent with a gap parameter of $\mathrm{d}_{x^{2}-y^{2}}$ wave type.
\end{abstract}

Keywords: Specific heat; Van Hove singularity; Gap parameter symmetry

\section{Introduction}

High resolution angle-resolved photoemission data on high- $T_{\mathrm{c}}$ superconductors (HTS) [1-3] have recently allowed to identify the presence of a saddle point in the band structure corresponding to a logarithmic Van Hove singularity (VHS) located in the density of states within $100-200 \mathrm{~K}$ of the Fermi energy.

The effect of such a singularity in the BCS theory [4] is known to allow for an increase of the critical temperature $T_{\mathrm{c}}$ to about $100 \mathrm{~K}[5-7]$ and to explain several properties of high- $T_{\mathrm{c}}$ cuprates like the doping dependence of $T_{\mathrm{c}}$ [5] and the linear temperature behavior of the in-plane resistivity $\rho_{a b}$ [7].

On the other hand, Dagotto et al. [8] have recently

\footnotetext{
* Corresponding author. Fax: +3241662990.

'E-mail: dorbolo@gw.unipc.ulg.ac.be.

${ }^{2}$ E-mail: houssa@gw.unipc.ulg.ac.be.

${ }^{3}$ E-mail: ausloos@gw.unipc.ulg.ac.be.
}

proposed a theoretical model including both the effect of a VHS and antiferromagnetic fluctuations which might be responsible for the formation of Cooper pairs and which allows one to explain the main features of high- $T_{\mathrm{c}}$ materials [8]. This model predicts a gap parameter of $\mathrm{d}_{x^{2}-y^{2}}$ wave type, the symmetry of which seems the most probable one in the cuprates [9-12].

One of the main properties to be investigated in superconducting materials is the specific heat [13] near $T_{c}$ or at low temperature. In this paper, we analyse the influence of a VHS on the temperature dependence of the electronic component of the specific heat $C_{\mathrm{e}}$ for a 2D superconductor. We consider both isotropic s-wave and anisotropic d-wave gap parameters as seem to be the alternative in HTS. The specific heat jump at $T_{\mathrm{c}}$ is reproduced in both models but the analysis of the very low temperature behavior of $C_{\mathrm{e}}$ in a single crystal of $\mathrm{YBa}_{2} \mathrm{Cu}_{3} \mathrm{O}_{7-\delta}$ [14] is only consistent with an anisotropic d-wave gap. 
The theoretical model is described in Section 2. In Section 3 , we compare the results obtained for respectively s-wave and d-wave gap parameters and discuss the very low temperature behavior of $C_{\mathrm{e}}$ in a single crystal of $\mathrm{YBa}_{2} \mathrm{Cu}_{3} \mathrm{O}_{7-\delta}$ [14]. Conclusions are finally drawn in Section 4 .

\section{Theoretical model}

The electronic specific heat $C_{\mathrm{e}}$ of a superconductor is calculated using the following expression [15]

$C_{\mathrm{e}}=\frac{\mathrm{d}}{\mathrm{d} T} \int \mathrm{d} \boldsymbol{k} E(\boldsymbol{k}) f(\boldsymbol{k})$,

where $E(k)=\sqrt{\left(\varepsilon-\varepsilon_{\mathrm{F}}\right)^{2}+\Delta(k)^{2}}$ is the quasiparticle energy spectrum near the Fermi energy $\varepsilon_{\mathrm{F}}$, and $f(k)=1 /\left[1+\exp \left(E / k_{\mathrm{B}} T\right)\right]$ is the Fermi-Dirac distribution function.

We consider a two-dimensional band structure with a saddle point located at the Fermi level. This electronic spectrum is given by $[5,7]$

$\varepsilon(\boldsymbol{k})-\varepsilon_{\mathrm{F}}=\left(\hbar^{2} / 2 m^{*}\right) k_{x} k_{y}$,

where $m^{*}$ is the effective mass of electrons in the $a b\left(\mathrm{CuO}_{2}\right)$ plane. The spectrum described by Eq. (2) gives a logarithmic singularity in the density of states at the Fermi energy $N(\varepsilon)=(1 / D) \ln \left|\varepsilon_{\mathrm{F}}\right|$ $\left(\varepsilon-\varepsilon_{\mathrm{F}}\right) \mid$ where $D$ is the bandwidth $[5,7]$.

We consider two different symmetries for the gap parameter, i.e. an isotropic s-wave gap parameter [13]

$\Delta_{\mathrm{s}}(T)=\Delta(0) \tanh \left(\alpha \sqrt{\left(T_{\mathrm{c}}-T\right) / T}\right)$,

where $\Delta(0)$ is the superconducting energy gap at zero temperature and $\alpha \approx 2$, and an anisotropic $\mathrm{d}_{x^{2}-y^{2}}$ wave gap parameter $[9,16]$

$$
\begin{aligned}
\Delta_{\mathrm{d}_{x^{2} y^{2}}} & (\boldsymbol{k}, T) \\
= & \Delta(0)\left[\cos \left(\hat{k}_{x}\right)-\cos \left(\hat{k}_{y}\right)\right] \\
& \times \tanh \left(\alpha \sqrt{\left(T_{\mathrm{c}}-T\right) / T}\right)
\end{aligned}
$$

where $\hat{k}_{x}=k_{x} a$ and $\hat{k}_{y}=k_{y} b$, with $a(b)$ the crystal parameter along the $a(b)$ axis. In the next section, we compare the numerical results obtained for both gap parameter symmetries.

\section{Results and discussion}

\section{1. s-wave gap parameter}

The temperature dependence of the electronic specific heat $C_{\mathrm{e}}$ for the isotropic s-wave gap parameter

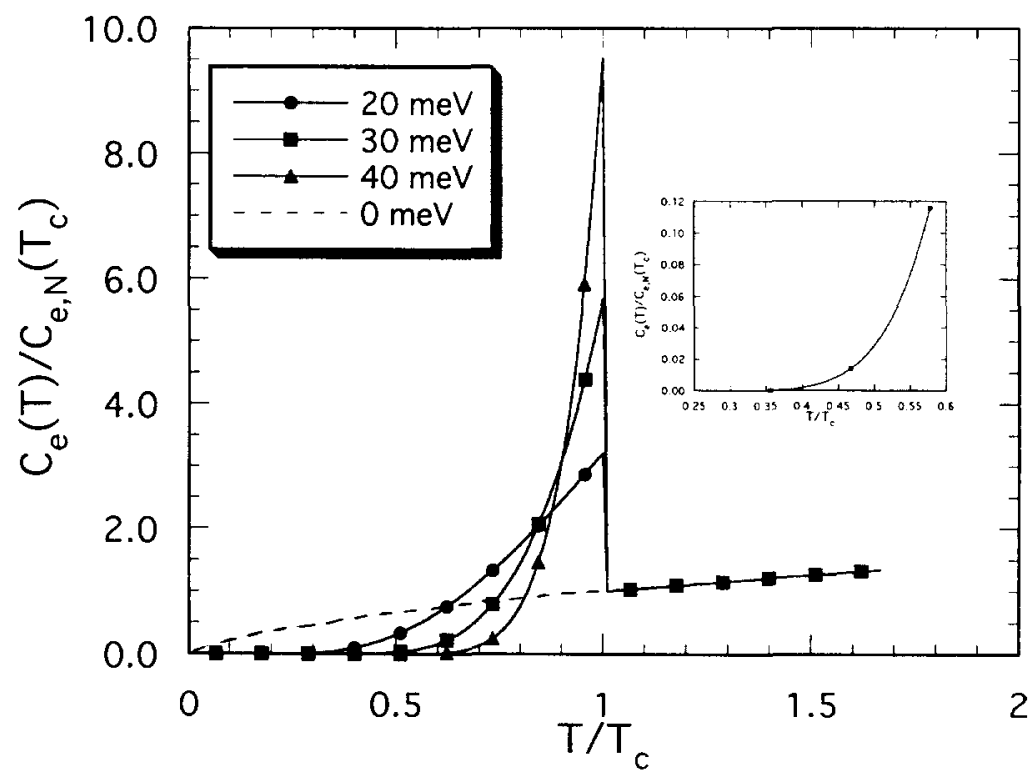

Fig. 1. Normalized electronic specific heat $C_{e} / C_{e . N}\left(T_{c}\right)$ versus reduced temperature $T / T_{\mathrm{c}}$ for the s-wave gap parameter as a function of the zero temperature gap $\Delta(0)$. Inset: low exponential temperature behavior of $C_{\mathrm{e}} / C_{\mathrm{e}, \mathrm{N}}\left(T_{\mathrm{c}}\right)$ for an s-wave gap. 


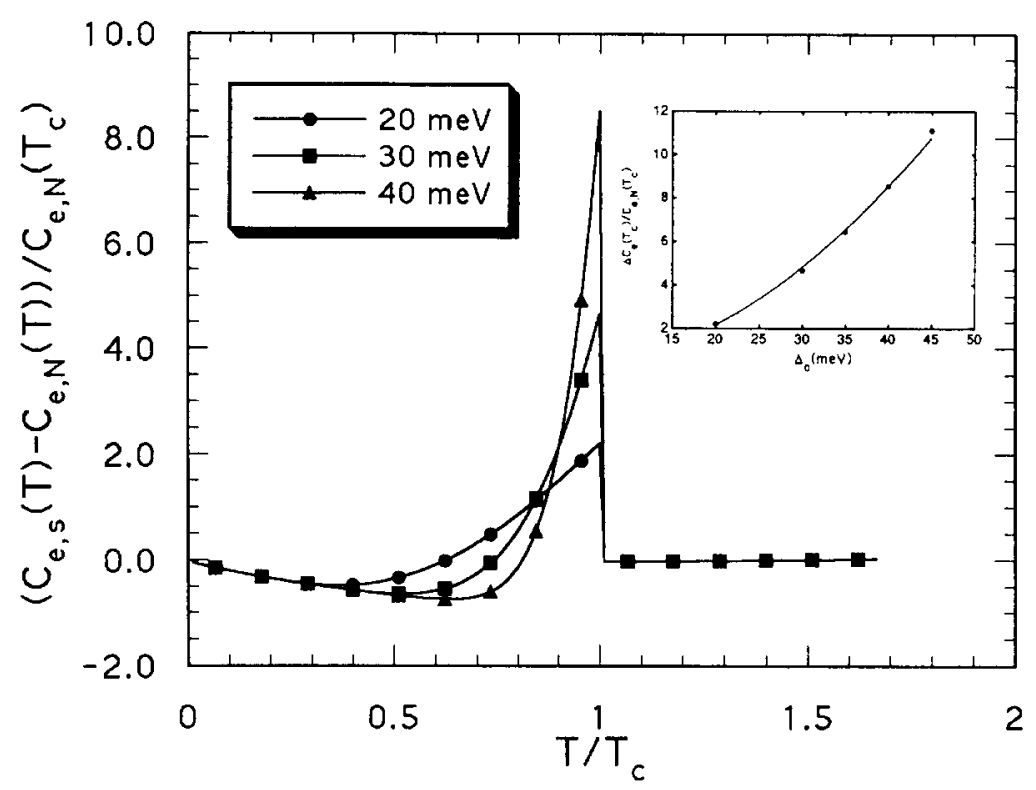

Fig. 2. Temperature dependence of the normalized electronic specific heat difference $\Delta C_{\mathrm{e}} / C_{\mathrm{e}, \mathrm{N}}\left(T_{\mathrm{c}}\right)=\left(C_{\mathrm{e}, \mathrm{s}}-C_{\mathrm{e}, \mathrm{N}}\right) / C_{\mathrm{e}, \mathrm{N}}\left(T_{\mathrm{c}}\right)$ in the s-wave case as a function of $\Delta(0)$. Inset: $\Delta C_{e} / C_{e, N}\left(T_{c}\right)$ as a function of $\Delta(0)$.

Eq. (3) is shown in Fig. 1 as a function of the reduced temperature $T / T_{\mathrm{c}}$ and for different zero temperature energy gaps $\Delta(0)$. One can see that the jump at $T_{\mathrm{c}}$ for this $2 \mathrm{D}$ system increases with $\Delta(0)$ and is of the order of $\Delta C_{\mathrm{e}}\left(T_{\mathrm{c}}\right) / C_{\mathrm{e}, \mathrm{N}}\left(T_{\mathrm{c}}\right) \approx 4$ for $\Delta(0)=30 \mathrm{meV}$ compared to the well-known BCS universal value of 1.43 [13] in 3D. At low temperatures, $C_{\mathrm{e}}$ decreases exponentially, see inset of Fig. 1.

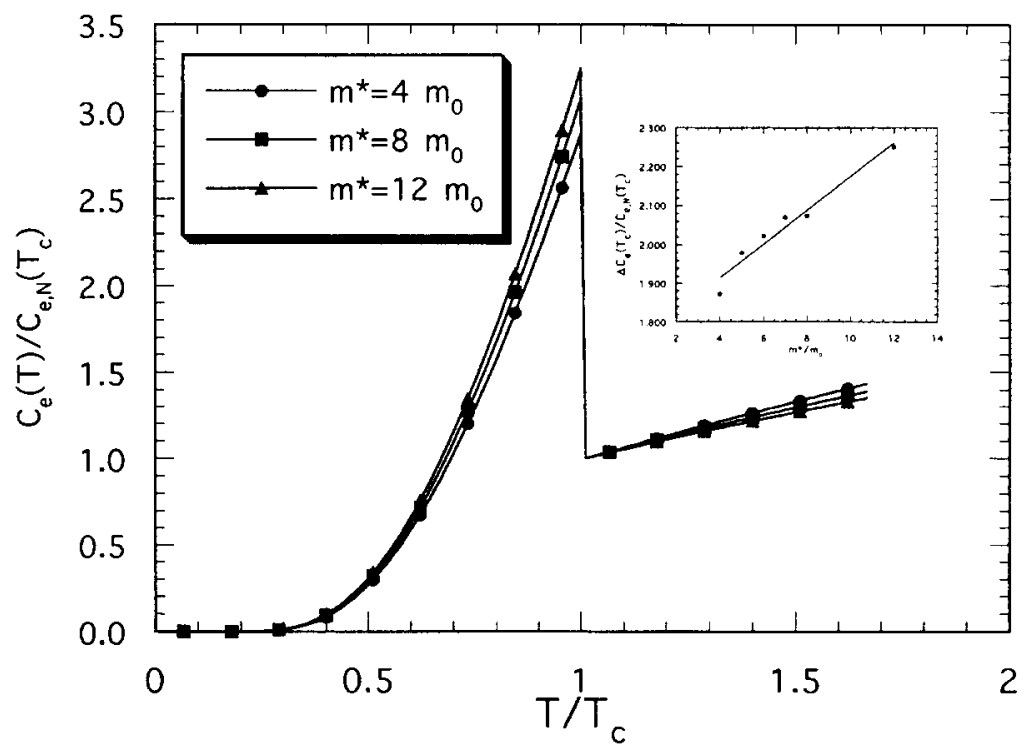

Fig. 3. Influence of the effective mass $m^{*}$ on the temperature dependence of $C_{\mathrm{e}} / C_{\mathrm{e}, \mathrm{N}}\left(T_{\mathrm{c}}\right)$ for the s-wave gap parameter. Inset: electronic specific heat jump at $T_{\mathrm{c}} \Delta C_{\mathrm{e}}\left(T_{\mathrm{c}}\right)$ versus $m^{*}$. 
This is known in 3D systems as being due to the exponential decrease of the number of quasiparticles characteristic of an s-wave gap parameter [13]. The same argument goes for 2D systems.

The specific heat difference $\Delta C_{\mathrm{e}}(T)=C_{\mathrm{e}, \mathrm{s}}(T)-$ $C_{\mathrm{e}, \mathrm{N}}(T)$, plotted in Fig. 2, changes sign between $T=0.6-0.8 T_{\mathrm{c}}$, depending on the value of $\Delta(0)$. Notice that the 3D BCS prediction for $\Delta C_{\mathrm{e}}(T)=0$ is $0.55 T_{\mathrm{c}}[13]$.

The influence of $\Delta(0)$ on the specific heat jump at $T_{\mathrm{c}}$ is plotted in the inset of Fig. 2. We observe that $\Delta C_{\mathrm{e}} \propto(\Delta(0))^{2}$ for an s-wave gap parameter.

The effect of the Van Hove singularity on the electronic specific heat of an s-wave superconductor, namely the increase of the value of the specific heat jump at $T_{c}$ and the increase of the temperature at which there is a sign change of the specific heat difference $\Delta C_{\mathrm{e}}$ compared to the BCS predictions are in agreement with experimental data on $\mathrm{YBa}_{2} \mathrm{Cu}_{3}$ $\mathrm{O}_{7-\delta}$ for which $\Delta C\left(T_{\mathrm{c}}\right) / C_{\mathrm{N}}\left(T_{\mathrm{c}}\right) \approx 4.8$ and $\Delta C(T)$ $=0$ at $T=0.82 T_{\mathrm{c}}$ [17]. However, we stress here that $\mathrm{YBa}_{2} \mathrm{Cu}_{3} \mathrm{O}_{7-\delta}$ is one of the least layered high- $T_{c}$ materials and consequently the strictly two dimensional spectrum given by Eq. (2) could be not quite appropriate for discussing features in this material.
A test of the quantitative finding stability with respect to parameter changes is made by varying the effective mass. The effect of the electron mass $m^{*}$ variation on $C_{\mathrm{c}}(T)$ is shown in Fig. 3. The jump at $T_{c}$ is decreased when the effective mass decreases. From the inset of Fig. 3, one can see that $\Delta C_{\mathrm{e}}\left(T_{\mathrm{c}}\right) \propto$ $\left(m^{*} / m_{0}\right)$.

\section{2. $d_{x^{2}-y^{2}}$ wave gap parameter}

The electronic specific heat $C_{\mathrm{e}}$ versus the reduced temperature $T / T_{\mathrm{c}}$ for the d-wave gap parameter case is presented in Fig. 4 for several values of $\Delta(0)$. The specific heat jump at $T_{\mathrm{c}}$ is of the order $\Delta C_{\mathrm{e}}\left(T_{\mathrm{c}}\right) / C_{\mathrm{e}, \mathrm{N}}\left(T_{\mathrm{c}}\right) \approx 1.8$ for $\Delta(0)=40 \mathrm{meV}$ and is smaller than in the s-wave case. This is probably because there are a few more non-superconducting particles when a d-wave gap parameter opens compared to an s-wave gap. The jump magnitude is in fine agreement with experimental data on $\mathrm{Bi}_{2} \mathrm{Sr}_{2}$ $\mathrm{CaCu}_{2} \mathrm{O}_{8}$ [18-20] which is much more two dimensional than $\mathrm{YBa}_{2} \mathrm{Cu}_{3} \mathrm{O}_{7-\delta}$ indeed. Experimental data gives a smaller specific heat discontinuity of the order of $\Delta C\left(T_{\mathrm{c}}\right) / C_{\mathrm{N}}\left(T_{\mathrm{c}}\right) \approx 1.3-1.5[18-20]$.

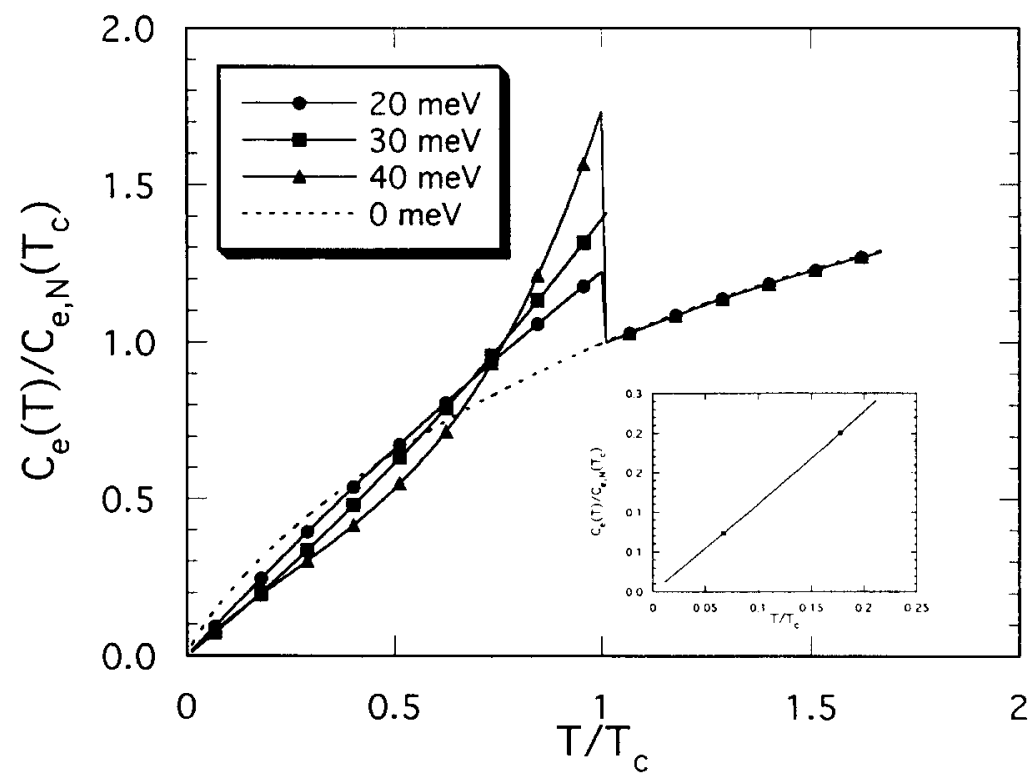

Fig. 4. Normalized electronic specific heat $C_{\mathrm{e}} / C_{\mathrm{e}, \mathrm{N}}\left(T_{\mathrm{c}}\right)$ versus reduced temperature $T / T_{\mathrm{c}}$ for the d-wave gap parameter as a function of $\Delta(0)$. Inset: low linear temperature behavior of $C_{e} / C_{e, N}\left(T_{\mathrm{c}}\right)$ for a d-wave gap. 


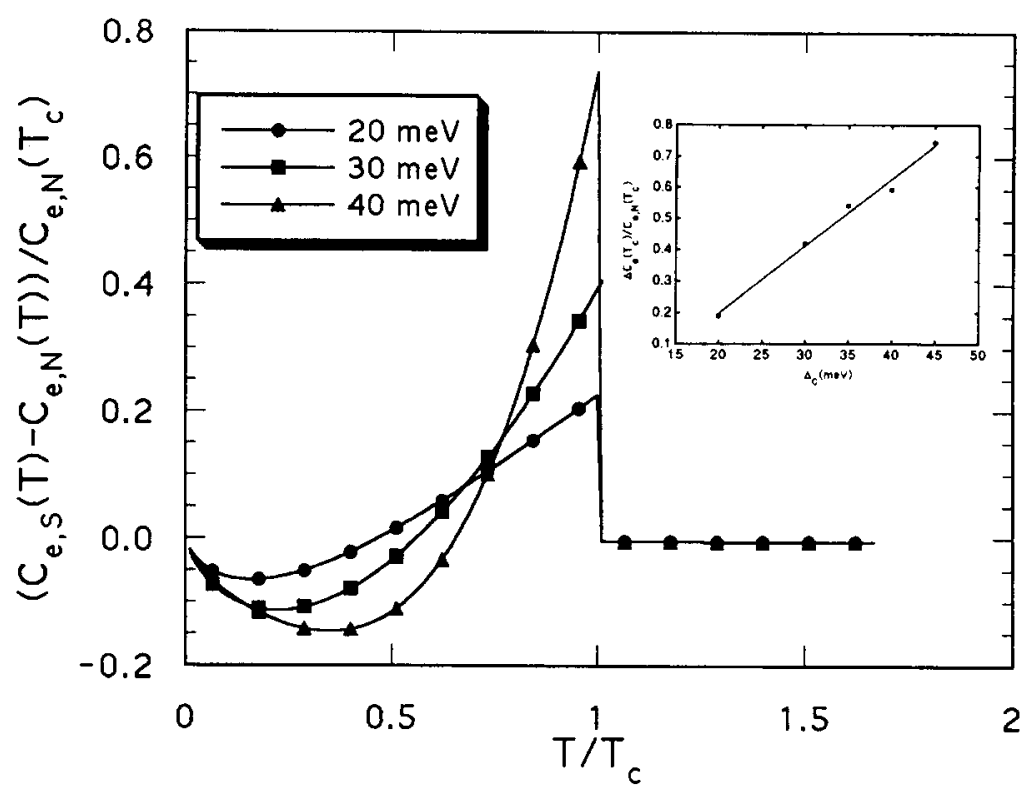

Fig. 5. Temperature dependence of the normalized electronic specific heat difference $\Delta C_{\mathrm{e}} / C_{\mathrm{e} . \mathrm{N}}\left(T_{\mathrm{c}}\right)=\left(C_{\mathrm{e}, \mathrm{s}}-C_{\mathrm{e}, \mathrm{N}}\right) / C_{\mathrm{e}, \mathrm{N}}\left(T_{\mathrm{c}}\right)$ in the d-wave case as a function of $\Delta(0)$. Inset: $\Delta C_{\mathrm{e}} / C_{\mathrm{e}, \mathrm{N}}$ versus $\Delta(0)$.

The low temperature behavior of $C_{\mathrm{e}}$ is quite different from the s-wave case: $C_{\mathrm{e}}$ follows approximately a linear temperature behavior (see inset of
Fig. 4). This is attributed to the presence of low energy excitations characteristic of a superconducting gap with lines of nodes.

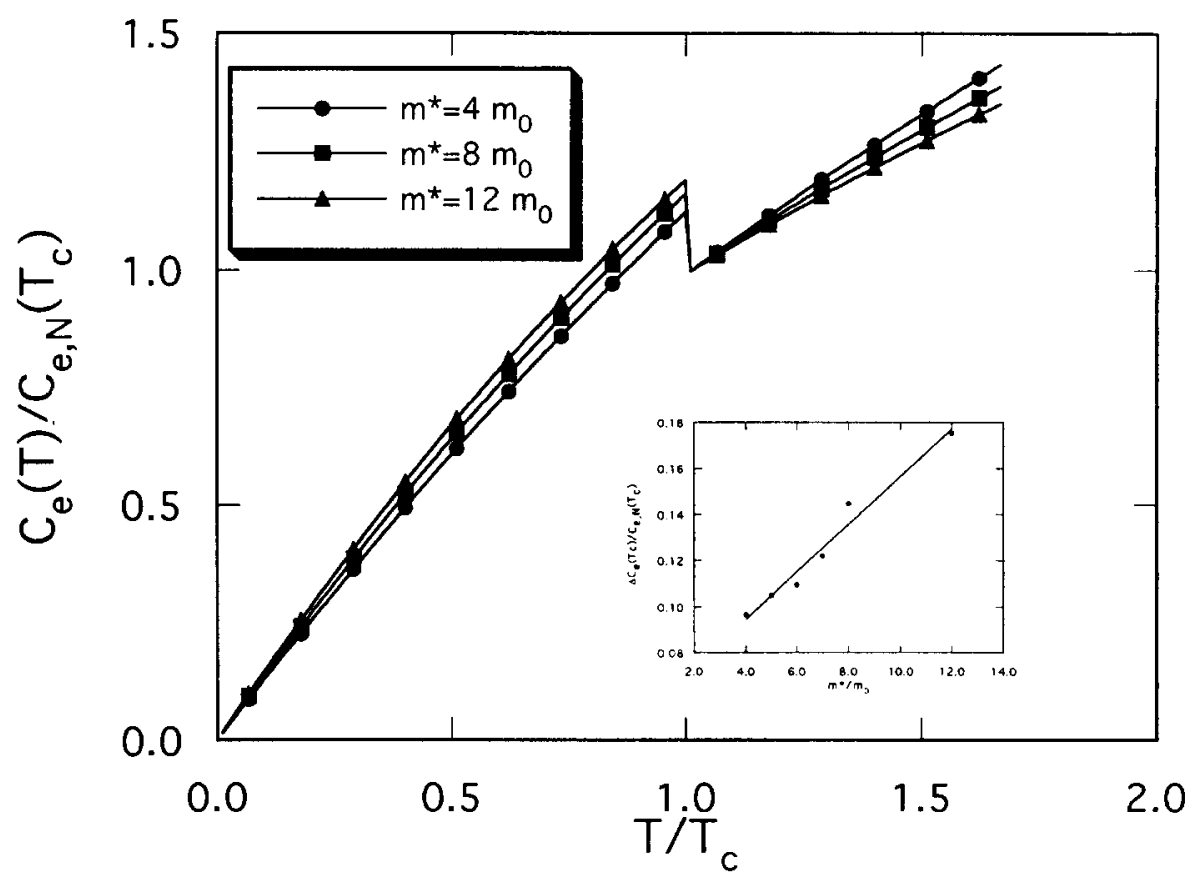

Fig. 6. Influence of the effective mass $m^{*}$ on the temperature dependence of $C_{\mathrm{e}} / C_{\mathrm{e}, \mathrm{N}}\left(T_{\mathrm{c}}\right)$ for the d-wave gap parameter. Inset: electronic specific heat jump at $T_{\mathrm{c}} \Delta C_{\mathrm{e}}\left(T_{\mathrm{c}}\right)$ versus $m^{*}$. 
The temperature dependence of the specific heat difference $\Delta C_{\mathrm{e}}$ is of interest and shown in Fig. 5. One observes that $\Delta C_{\mathrm{e}}$ crosses the temperature axis at about $0.5-0.6 T_{c}$, depending on the value of $\Delta(0)$. This lower temperature range where a change of sign occurs does not seem to have been investigated in quasi two dimensional materials like $\mathrm{Bi}_{2} \mathrm{Sr}_{2} \mathrm{CaCu}_{2} \mathrm{O}_{8}$ (see Section 4 for comments).

The specific heat jump at $T_{\mathrm{c}}$ is found to increase linearly with $\Delta(0)$ for a d-wave gap parameter, see the inset of Fig. 5.

The influence of the effective mass $m^{*}$ variation on $C_{\mathrm{e}}(T)$ is presented in Fig. 6. Like in the s-wave case, we observe that $\Delta C_{\mathrm{e}}\left(T_{\mathrm{c}}\right)$ decreases when the effective mass is lowered and behaves approximately like $\Delta C_{\mathrm{e}}\left(T_{\mathrm{c}}\right) \propto\left(\mathrm{m}^{*} / \mathrm{m}_{0}\right)$, cf. the inset of Fig. 6 .

\subsection{Low temperature behavior of $C_{e}$}

The very low temperature behavior of the total specific heat $C(T)$ is of interest. The case of a single crystal of $\mathrm{YBa}_{2} \mathrm{Cu}_{3} \mathrm{O}_{7-\delta}$ [14] is shown in Fig. 7a. $C(T)$ can be usually separated into two main contributions, namely a phonon contribution $C_{\mathrm{ph}}$ which behaves like $T^{3}$ and an electronic contribution $C_{\mathrm{e}}$ markedly depending on the electronic features, i.e.,

$$
C(T)=\beta T^{3}+C_{\mathrm{e}}(T) .
$$

The phonon contribution can be obtained by analysing the magnetic field behavior of $C(T, B)$ assuming that this contribution is not altered by the magnetic induction [14,21]. The authors of Ref. [14] then obtain $\beta=0.392 \mathrm{~mJ} / \mathrm{mol} \mathrm{K}^{4}$. The electronic contribution $C_{\mathrm{e}}(T)$ obtained by substracting this phonon background is shown in Fig. $7 \mathrm{~b}$ on a $\log -\log$ plot. $C_{\mathrm{e}}(T)$ behaves almost linearly with temperature, in fine agreement with the above theoretical prediction for a d-wave gap parameter in presence of a Van Hove singularity. Notice also that this linear term should be absent if the gap parameter was of isotropic s-wave type since $C_{\mathrm{e}}$ decreases exponentially for such a gap even in the presence of a VHS, see Fig. 1.

We point out that Moler et al. [14] introduced an extra $T^{2}$ term in $C_{\mathrm{e}}(T)$ in order to fit their data and claimed that such a $T^{2}$ term might arise from a $\mathrm{d}$-wave gap parameter. We did not find here any evidence for the need of a $T^{2}$ and from our theoreti-
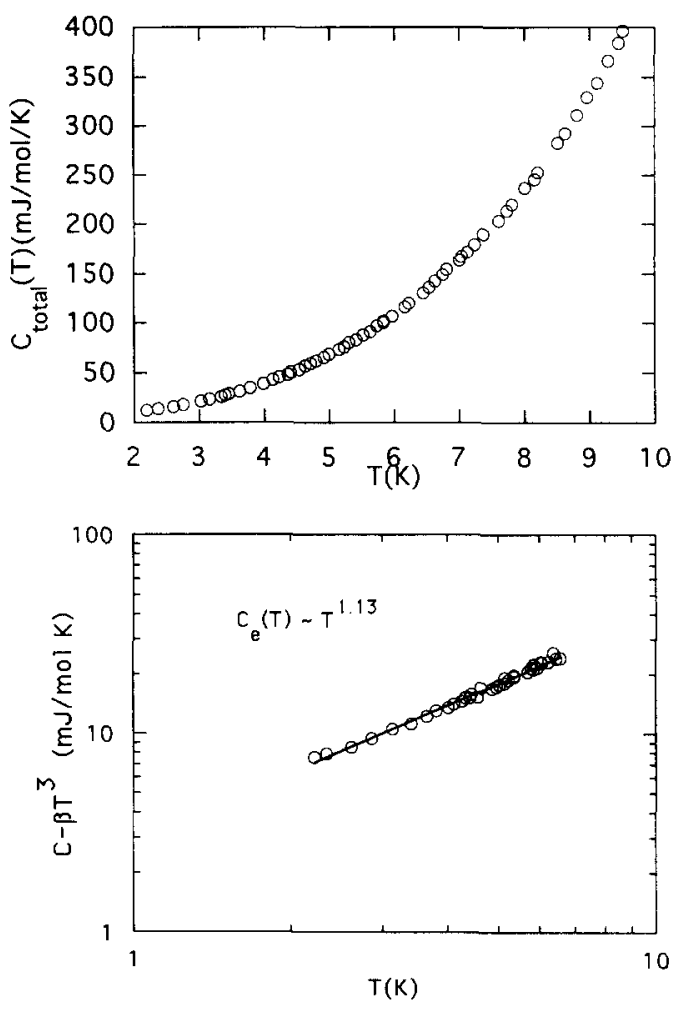

Fig. 7. (a) Temperature dependence of the specific heat of a single crystal of $\mathrm{YBa}_{2} \mathrm{Cu}_{3} \mathrm{O}_{7-\delta}$ from Ref. [11]. (b) Temperature dependence of the electronic specific heat $C_{\mathrm{e}}(T)=C(T)-\beta T^{3}$ of a single crystal of $\mathrm{YBa}_{2} \mathrm{Cu}_{3} \mathrm{O}_{7-8}$ from data in Ref. [11].

cal results, the effect of a VHS on a d-wave superconductor does not seem to give a $T^{2}$ term.

Notice also that there seems to be no need for the Schottky $T^{-2}$ term in absence of magnetic field and for such a pure single crystal.

Finally, it is worth to mention that Junod et al. [22] recently analysed the very low temperature behavior of the specific heat of a single crystal of $\mathrm{Bi}_{2} \mathrm{Sr}_{2} \mathrm{CaCu}_{2} \mathrm{O}_{8}$. These authors also found a quasi linear electronic contribution $C_{\mathrm{e}} \propto T$, in agreement with our findings for a two dimensional d-wave superconductor.

\section{Conclusions}

In this work, we have analysed the influence of a logarithmic Van Hove singularity on the electronic specific heat of a two dimensional superconductor. The experimental results in high- $T_{\mathrm{c}}$ superconductors, 
mainly the specific heat jump at $T_{\mathrm{c}}$ and the temperature crossover of $\Delta C_{\mathrm{e}}$ can be explained by considering either isotropic s-wave or anisotropic d-wave gap parameters. Notice however that the experimental results on $\mathrm{Bi}_{2} \mathrm{Sr}_{2} \mathrm{CaCu}_{2} \mathrm{O}_{8}$ for which our two dimensional model should be quite appropriate, can be better described by considering a d-wave gap parameter with reasonable values of the physical parameters, i.e. a zero temperature gap parameter $\Delta(0) \approx 30$ $\mathrm{meV}$ [23] and an effective mass $\mathrm{m}^{*}$ of the order of $8 m_{0}$ [24].

Furthermore, the very low temperature behavior of the electronic specific heat of a single crystal of $\mathrm{YBa}_{2} \mathrm{Cu}_{3} \mathrm{O}_{7-\delta}$ is incompatible with an isotropic $\mathrm{s}$-wave gap parameter. This strongly supports the d-wave scenario in high- $T_{c}$ compounds.

As a final point we should mention that the $\Delta C=0$ value consists in an experimental point of interest in more complex parameter fits. It is also clear that the band structure of such HTS is more complex than Eq. (2). However, we believe that the overall temperature dependence will not be modified by using more complex spectra (quantitative values of the parameters might be modified). More self consistent calculations will be now of interest, together with new data analysis in the vicinity of the critical temperature for extracting the critical fluctuations. It is known indeed in such an endeavour that the correct background should be extracted. A d-wave mean field approximation is thus necessary at first.

Finally, we received a paper from Newns et al. [25] during the preparation of this manuscript. These authors have studied the influence of a VHS on various features, among them being the specific heat jump of a d-wave superconductor and they have found similar results as ours for that case.

\section{Ackowledgements}

Part of this work has been financially supported through the Impulse Program on High Temperature Superconductors of Belgium Federal Services for Scientific, Technological and Cultural (SSTC) Affairs under contract SU/02/013 and the ARC 94-99/174 contract of the Ministery of Higher Education and Scientific Research through the University of Liège Research Council.

\section{References}

[1] D.S. Dessau, Z.X. Shen, D.M. King, D.S. Marshall, L.W. Lombardo, P.H. Dickinson, A.G. Loeser, J. DiCarlo, C.H. Park, A. Kapitulnik and W.E. Spicer, Phys. Rev. Lett. 71 (1993) 2781 .

[2] K. Gofron, J.C. Campuzano, A.A. Abrikosov, M. Lindroos, A. Bansil, H. Ding, D. Koelling and B. Dabrowski, Phys. Rev. Lett. 73 (1994) 3302.

[3] J. Ma, C. Quitmann, R.J. Kelley, P. Alméras, H. Berger, G. Margaritondo and M. Onellion, Phys. Rev. B 51 (1995) 3832.

[4] G. Rickayzen, in: Superconductivity, Vol. 1, ed. R.D. Parks (M. Dekker, New York, 1969) p. 51.

[5] D.M. Newns, C.C. Tsuei, P.C. Pattnaik and C.L. Kane, Commun. Condens. Matter Phys. 15 (1992) 273.

[6] A. Gama Goicochea, Phys. Rev. B 49 (1994) 6864.

[7] D.M. Newns, C.C. Tsuei, R.P. Huebener, P.J.M. van Bentum, P.C. Pattnaik and C.C. Chi, Phys. Rev. Lett. 73 (1994) 1695 .

[8] E. Dagotto, A. Nazarenko, and A. Moreo, Phys. Rev. Lett. 74 (1995) 310.

[9] D.J. Scalapino, Phys. Rep. 250 (1995) 329.

[10] A. Sudbø and S.P. Strong, Phys. Rev. B 51 (1995) 1338.

[11] F. Onufrieva, Physica C 251 (1995) 348.

[12] M. Houssa and M. Ausloos, Europhys. Lett. 33 (1996) 695.

[13] R. Meservey and B.B. Schwartz, in: Superconductivity, Vol. 1, ed. R.D. Parks (M. Dekker, New York, 1969) p. 117.

[14] K.A. Moler, D.J. Baar, J.S. Urbach, R. Liang, W.N. Hardy and A. Kapitulnik, Phys. Rev Lett. 73 (1994) 2744.

[15] A.A. Abrikosov, Introduction to the Theory of Normal Metals, Solid State Physics (suppl. 12) (Academic Press, New York, 1972) p. 31

[16] J.H. Xu, J.L. Shen, J.H. Miller and C.S. Ting, Phys. Rev. Lett. 73 (1994) 2492.

[17] A. Junod, A. Bezinge, D. Eckert, T. Graf and J. Müller, Physica C 152 (1988) 495.

[18] A.K. Bandyopadhyay, P. Maruthikumar, G.L. Bhalla, S.K. Agarwal and A.V. Narlikar, Physica C 165 (1990) 29.

[19] E. Braun, W. Schnelle, H. Broicher, J. Harnischmacher, D. Wohlleben, C. Allgeier, W. Reith, J.S. Schilling, J. Bock, E. Preisler and G.J. Vogt, Z. Phys. B 84 (1991) 333.

[20] W. Schnelle, N. Knauf, J. Bock, E. Preisler and J. Hüdepohl, Physica C 209 (1993) 456.

[21] N. Overemd, M.A. Howson, and I.D. Lawrie, Phys. Rev. Lett. 72 (1994) 3238;

M. Roulin, A. Junod and J. Müller, Phys. Rev. Lett. 75 (1995) 1869.

[22] A. Junod, K.Q. Wang, T. Tsukamoto, B. Revaz, G. Triscone, E. Walker and J. Muller, Physica C 235-240 (1994).

[23] R. Koltun, M. Hoffmann, P.C. Splittgerber-Hünnekes and G. Güntherodt, Physica B 165-166 (1990) 1567.

[24] D.R. Harshman and A.P. Mills, Phys. Rev. B 45 (1992) 10684.

[25] D.M. Newns, C.C. Tsuei and P.C. Pattnaik, Phys. Rev. B 52 (1995) 13611. 